\title{
jLiberen a los estudiantes de su disciplina! \\ La formación de los arquitectos en la era 2.0
}

Free students from their discipline! Architects' academic training in the 2.0 era

\author{
> Alejandro Ariel Moreira \\ Centro de Informática y Diseño \\ Facultad de Arquitectura, Diseño y Urbanismo. \\ Universidad Nacional del Litoral, Argentina \\ amoreira@fadu.unl.edu.ar
}

\author{
> Cecilia Parera \\ Centro de Informática y Diseño \\ Facultad de Arquitectura, Diseño y Urbanismo. \\ Universidad Nacional del Litoral, Argentina \\ cparera@fadu.unl.edu.ar
}

\begin{abstract}
In the context of recent transformations in the professional practice of architecture after the incorporation of new developments in digital technology, the rigid disciplinary and scope of intervention delimitations that characterize most academic proposals have lost support. This paper intends to contribute, in general, to the consideration about the referred problem, and, in particular, to the discussion about the urge to review the curricula of the architectural programs of most Latin-American universities, still defined by the concept of an autonomous and individual architect.
\end{abstract}

Keywords: Diseńo colaborativo, Innovaciones tecnológicas, Enseñanza de la arquitectura

\section{Introducción}

La definición de la arquitectura como profesión y disciplina independiente que actualmente rige los planes de estudio de la mayoría de las instituciones de formación de arquitectos en Iberoamérica se remonta a conceptualizaciones inicialmente planteadas por el humanismo renacentista del siglo XV (Carpo, 2009). Particularmente a partir de las consideraciones expresadas por Leon Battista Alberti en su libro De re aedificatoria (1450) es que la incumbencia de los arquitectos fue recortada al ámbito del proyecto, transformándose en intelectuales creadores que, en forma autónoma respecto de otros campos de conocimiento desarrollaban un proyecto en su totalidad, entregando a los constructores dibujos y maquetas para que éstos los ejecutaran sin introducir cambios. En consecuencia, este nuevo rol también trajo consigo una profunda división del trabajo, distanciando a los arquitectos de carpinteros, albañiles y constructores, entre tantos otros profesionales vinculados con la industria de la construcción.

Este paradigma disciplinar, rubricado en tratados posteriores y consolidado con el modelo de formación de las Academias y de L'École des Beaux Arts de París, no sufrió sustanciales modificaciones ni en la propuesta de instituciones que incorporaron el rigor técnico del modelo de L'Ecole Polytechnique de Paris, ni ante los significativos cambios planteados por la Revolución Industrial. En este contexto, los arquitectos sólo se sintieron forzados a realizar una mayor cantidad de representaciones bi y tridimensionales, y a contactar especialistas que los asesoraran en cuestiones técnicas puntuales a medida que la complejidad y la escala de los proyectos de arquitectura aumentaba. Si bien en las últimas décadas del siglo XX la introducción de herramientas de Computer Aided Design (CAD) logró optimizar los tiempos y costos involucrados en el desarrollo de los proyectos y en los medios de representación, no lograron transformar la naturaleza autónoma y fragmentada de la práctica profesional.

Sin embargo, la reciente introducción en el campo de la arquitectura de nuevos desarrollos en tecnología digital asociados a los conceptos de Knowledge Management-proceso de captura, análisis, intercambio y utilización de información entre miembros de un equipo profesional- e Integrated Practice-práctica profesional caracterizada por plantear un proceso colaborativo entre expertos de diversas disciplinas para el desarrollo de sus proyectos- han contribuido a cuestionar las bases de este modelo individual, particularmente a partir de herramientas que simplifican y optimizan la comunicación de las ideas aportadas por los distintos actores involucrados, como clientes, arquitectos, ingenieros civiles, economistas, representantes del Estado, constructores, fabricantes y supervisores de mantenimiento (Martin-Iglesias, 2011). La utilización de sistemas Building Information Modeling (BIM), entre tantas otras herramientas específicas o de uso generalizado disponibles en la era de la "digitalización cultural", ha posibilitado un trabajo colaborativo e interdisciplinario tanto durante la etapa de proyecto como la de construcción, contribuyendo a diluir el concepto de autoría individual y la división en las dos etapas anteriormente señaladas. 


\section{La enseñanza universitaria en el contexto profesional contemporáneo}

Si bien es posible afirmar que la apertura que muestra el ejercicio profesional constituye un significativo avance respecto de la tradicional definición de incumbencia de los arquitectos, resulta notorio que el modelo de enseñanza / aprendizaje que guía la formación de la mayoría de las universidades iberoamericanas aún se sustenta fuertemente en el modelo humanista. Es decir, el campo de la enseñanza se mantiene, en gran medida, inmune a las nuevas demandas del mercado, sin siquiera plantear de manera sistemática una trayectoria posible hacia este cuerpo de conocimientos. En consecuencia, los graduados son expertos en el abordaje individual del proceso de diseño de un proyecto arquitectónico, sin relacionarse directamente con otras cuestiones claves para su optimización. Salvo en el caso de experiencias académicas puntuales, generalmente dictadas bajo la modalidad de workshops y ofrecidas como asignaturas optativas, la construcción de respuestas proyectuales consensuadas, el procesamiento de grandes volúmenes de información, el intercambio colaborativo de conocimientos con otras disciplinas, y la adaptabilidad a contextos de prácticas disímiles, por nombrar algunas de las características de la práctica profesional contemporánea que son posibilitadas por los mencionados desarrollos en tecnología digital, no constituyen estrategias incluidas en los lineamientos pedagógicos de las instituciones que forman arquitectos, en general, o de las asignaturas que componen los planes de estudios, en particular (Cheng, 2006).

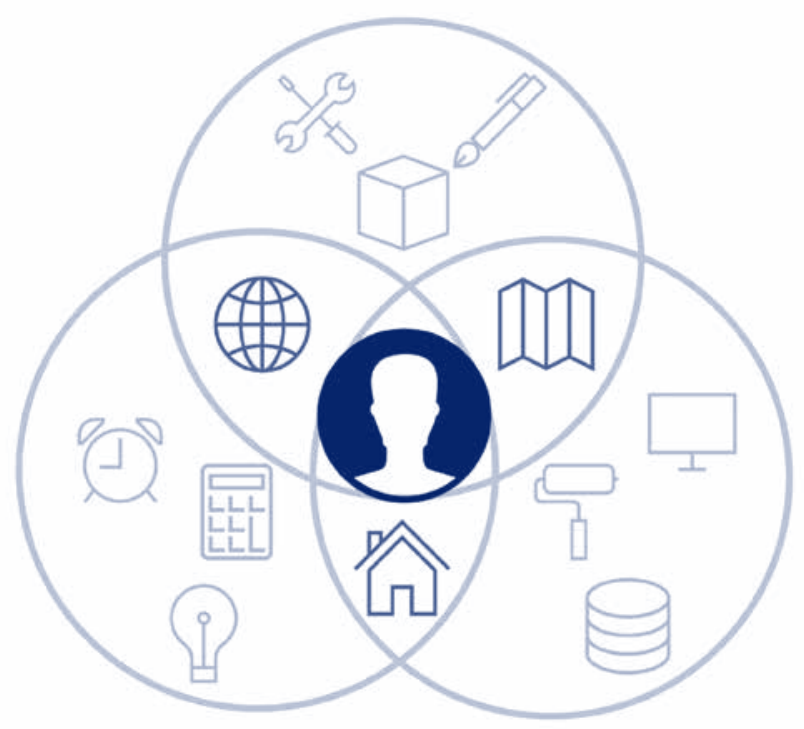

Figura l: Múltiples condicionamientos que indicen en la práctica profesional contemporánea.

Este contexto de notoria disparidad entre la práctica profesional contemporánea y la formación académica de los futuros arquitectos plantea la necesidad de repensar las estrategias que definen la enseñanza de la arquitectura, reconociendo que esta etapa constituye el inicio de un proceso de exploración y desarrollo que signará fuertemente la trayectoria profesional de sus egresados.
Los planes de estudios de las facultades de arquitectura latinoamericanas, en la generalidad de los casos, destinan la mitad de los créditos a asignaturas del área de diseño -liderada por los talleres de proyecto arquitectónico, pero también constituida por estudios morfológicos y urbanos-, respaldando el predominio del atelier como ámbito de formación por excelencia instaurado por el modelo formativo academicista en el siglo XVII. El resto de los créditos se distribuyen en "Estructuras", "Construcciones", "Instalaciones", "Historia", "Teoría” y "Práctica Profesional", por nombrar las denominaciones más frecuentemente identificadas en las otras áreas de conocimiento. El cuerpo de asignaturas electivas / optativas, por su misma categorización, tienden a ser asociadas con conocimientos no esenciales, al no ser obligatorias, y son asimilables en un número importante de ejemplos estudiados con algún sub-campo disciplinar, como sustentabilidad, domótica, luminotecnia, o bien la enseńanza de un software específico.

En el marco de este extendido planteo formativo, la pregunta inicial -casi podría decirse que existencial- es dónde introducir los nuevos desarrollos en tecnología digital que, como es el caso de los mencionados sistemas BIM, plantean incorporar una inédita estrategia proyectual y no sólo la enseñanza de un software. Como señala Peggy Deamer (2011), podrían ser ubicados en algún "Taller de Arquitectura”, ámbito directamente asociado con el desarrollo del ejercicio de diseño. También resultarían pertinentes en cursos de "Construcciones" o "Estructuras", para los que la manipulación de información sobre los materiales y sus características constituye una cuestión clave. Por su parte, en asignaturas tipo "Práctica Profesional" contribuirían con el entrenamiento de las destrezas que a futuro serán requeridas a los estudiantes en los estudios de arquitectura. Su presentación de manera independiente, como curso electivo, propiciaría un abordaje liberado de presiones por acoplarse a la modalidad definida en los lineamientos pedagógicos de la institución, las que muchas veces se encuentran atadas a conceptualizaciones tradicionales. Finalmente, su ofrecimiento como curso de posgrado plantearía la necesidad de incorporar un complemento a una formación de grado que, por falta de tiempo o convicción, no alcanza a cubrir las demandas vigentes en su propuesta obligatoria. La enumeración de asociaciones posibles podría continuar recorriendo cualquier plan de estudios; sin embargo, la diversidad de vinculaciones hasta aquí planteadas deja en evidencia el carácter integral de estos sistemas, para el que la división tradicional en sub-campos disciplinares constituye uno de los principales inconvenientes. Más aún, siendo que estos sistemas tienen su asidero en prácticas colaborativas y transdisciplinares, la misma autonomía disciplinar que estructura la enseñanza actual constituye un impedimento para el aprovechamiento cabal de sus beneficios.

\section{Un posible esquema formativo}

Reconociendo la necesidad de adecuar la oferta académica a la realidad de la práctica profesional contemporánea diversas experiencias pilotos han sido desarrolladas en los últimos ańos, particularmente en universidades norteamericanas, signadas por un contexto profesional en el que el uso de sistemas tipo BIM aumentó de un $28 \%$ 
en 2008 a 71\% en 2012 (Johnson, 2014). Tal es el caso del curso AEC web-based de Stanford University (Sanguinetti, 2009), del programa Master of Integrated Building Delivery ofrecido por el Illinois Institute of Technology (Durbrow \& Robertson, 2011), del curso Building Information Modeling for Commercial Construction dictado en Purdue University (Cory, 2012) o del taller Interdisciplinary Collaborative BIM Studio de Pennsylvania State University (Holland, Wing \& Goldberg, 2013).

Los resultados de estas propuestas permiten reconocer la necesidad de desarrollar en los estudiantes una doble capacidad. Por un lado, la de empatía con otras disciplinas, de manera que sean capaces de trabajar de manera colaborativa con el resto de los actores vinculados en el proceso de diseño, construcción, equipamiento y mantenimiento de las obras de arquitectura. Por otro lado, la del manejo de una amplia serie de conocimientos específicos que definen el campo de acción en el que tendrán que desarrollarse una vez que reciban sus títulos de arquitectos. A la hora de proponer un modelo pedagógico que satisfaga esta doble demanda, un esquema "en peine" -como el ilustrado en la Figura 2- pareciera resultar el más adecuado.

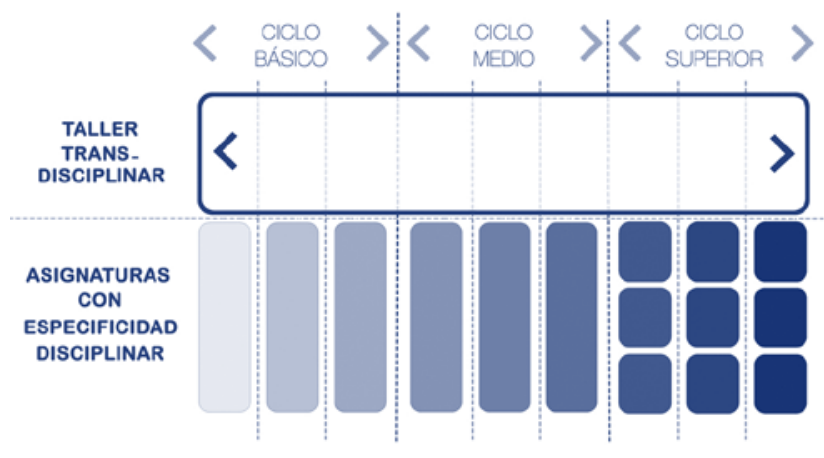

Figura 2: Esquema formativo propuesto.

Los tópicos que se intersectan en los distintos campos de conocimiento deberían ser abordados en cursos transdisciplinares anuales que mantienen las características del trabajo en taller. La transversalidad disciplinar, en este caso, permite la elaboración de conceptos y métodos nuevos, que no se ciñen estrictamente a una disciplina en particular (Whiting, 2003). En este ámbito, la aptitud más importante que debe ser estimulada por los docentes es la del pensamiento crítico, posibilitando la visualización simultánea de los múltiples aspectos asociados al problema planteado como ejercicio, así como sus relaciones. La adquisición / consolidación de esta capacidad excede la cuestión del medio utilizado -es decir, si es análogo o digital- y se vincula más bien a necesidades planteadas en el marco del concepto del Design Thinking (Dorst, 2011). Los desarrollos en tecnología digital son incorporados de manera progresiva, aumentando en complejidad a medida que el estudiante avanza en la carrera y ha desarrollado mayores niveles de sofisticación en sus conocimientos sobre principios de diseńo fundamentales, como forma, composición, jerarquía espacial y dimensión tectónica. En los años superiores, la manipulación de modelos digitales de almacenamiento de información, utilizando metodologías propuestas por los sistemas BIM, constituiría una de las instancias más avanzadas en la implementación de nuevas tecnologías.

Los "dientes" del esquema propuesto estarían constituidos por los conocimientos específicos requeridos por cada sub-campo, retomando el concepto que actualmente estructura las asignaturas que no pertenecen al área de diseño. Para asegurar la vinculación permanente con los talleres troncales anteriormente mencionados, cuestión fundamental para lograr una formación integral, se deben reforzar las estrategias de interacción entre todas las asignaturas del mismo año lectivo / ciclo formativo. Más aún, en los años superiores podrían ofrecerse líneas de especialización, planteadas como opciones profesionales definidas y no como una variedad de asignaturas electivas de asociación aleatoria. Estas orientaciones, por su parte, viabilizarían una flexibilidad en los planes de estudio acorde a la permanentemente cambiante realidad que define la práctica profesional contemporánea y los diferentes roles que sus egresados podrían asumir.

\section{Discusión}

Este trabajo avanza en una de las líneas de indagación planteadas en el Proyecto de Investigación CAI+D 2011 "El rol del arquitecto en la era de la digitalización cultural, ¿el fin del paradigma albertiano?", dirigido por los autores del presente trabajo y con sede en el Centro de Informática y Diseño de la Facultad de Arquitectura, Diseño y Urbanismo de la Universidad Nacional del Litoral, Argentina (FADU UNL). Un anticipo de reconocimiento de este inédito contexto fue presentado en SIGRADI 2012 (Moreira, 2012). Dado el carácter exploratorio de este artículo, no plantea respuestas definitivas sino ideas iniciales para la conceptualización de la temática, teniendo en mente la necesidad de una la revisión de los lineamientos pedagógicos y académicos que definen el proceso de enseñanza/aprendizaje de la arquitectura en la actualidad.

Como principal hipótesis se ha planteado que la implementación de los desarrollos en tecnología digital señalados con anterioridad no tardará en viralizarse en el contexto iberoamericano, tal como ha sucedido de manera vertiginosa en EE.UU., por lo que resulta imperioso anticipar transformaciones en el campo de la formación académica. Hace casi una década, al ser interrogado sobre las implicancias de las nuevas tecnologías en la práctica profesional de la arquitectura, el arquitecto Thom Mayne -cofundador del estudio Morphosis- indicó a los asistentes a la convención anual del American Institute of Architects que "si quieren sobrevivir, tendrán que cambiar. Si no cambian, perecerán. No ejercerán la arquitectura si no están a la velocidad de los cambios. Absolutamente no ejercerán en diez años". (Mayne, 2005, p. 5). El tiempo pasa, tomemos la posta.

\section{Bibliografia}

Carpo, M. (2009). Revolución 2.0. El fin de la autoría humanista.

En Revista Arquitectura Viva, n 124 (pp. 19-25). España.

Cheng, R. (2006). Questioning the role of BIM in architectural education. En AECbytes Viewpoint, n² 26, julio (pp. 1-5). 
Cory, C. (2012) Applying BIM in design curriculum. En N. Gu \& $\mathrm{X}$. Wang. Computational design methods and technologies. Applications in CAD, CAM and CAE education. Hershey, PA: IGI global.

Deamer, P. (2011). BIM in Academia. En Deamer, P. \& Bernstein, P. BIM in Academia (pp. 1-4). New Haven: Yale School of Architecture

Dorst, K. (2011). The core of 'design thinking' and its application. En Design Studies, volume 32, issue 7, November (pp. 521-532)

Durbrow, J.\& Robertson, D. (2011). Master of integrated building delivery: providing comprehensive delivery strategies to promote a quality built environment. En Deamer, P. \& Bernstein, P. BIM in Academia (pp. 100-106). New Haven: Yale School of Architecture.

Johnson, R. (2014) Building information modeling in construction education. En American Council for Construction Education Presentation. Tampa: Fl. Disponible en http://www.
acce-hq.org/images/uploads/Reid_Johnson_ACCE_Presentation-2014_Tampa.pdf

Holland, R., Wing, S. \& Goldberg, D. (2013). Interdisciplinary Collaborative BIM Studio. En BIM Academic Education Symposium. National Institute of Building Sciences. Washington D.C.

Martin-Iglesias, R. (2011). Hacia un nuevo paradigma de diseño colaborativo. En XV Congreso SIGRADI, FADU UNL, Santa Fe, Argentina (pp. 122-125).

Mayne, T. (2005). Change or perish. En American Institute of Architects National Convention, Las Vegas, EE.UU: AIA.

Moreira, A. (2012). Integración y colaboración tecnológica en arquitectura. En XVI Congreso SIGRADI, Fortaleza, Brasil (pp. 268-271).

Sanguinetti, P. (2009). BIM in academia: Shifting our attention from product to process. En Tidafi and T. Dorta (eds) Joining Languages, Cultures and Visions: CAADFutures (pp. 395-409).

Whiting, S. (2003). Going Public. En Hunch n 6/7, Berlage Institute Report (pp. 497-502). 